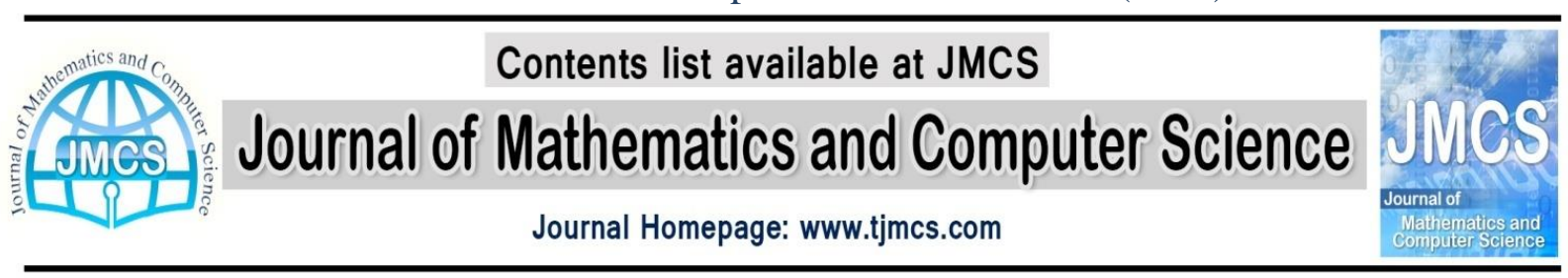

\title{
On Fuzzy Isomorphism Theorem Of Hypernear-modules
}

\author{
M. Aliakbarnia. Omran ${ }^{1}$, Y. Nasabi ${ }^{2}$, E. Hendukolaie ${ }^{3}$ \\ ${ }^{1}$ Amol Institution of higher education, Amol, Iran, \\ Mehdiomran@gmail.com \\ ${ }^{2}$ Young Researchers Club, Islamic Azad University, Ayatollah Amoli Branch, Amol, Iran, \\ Yaser.nasabi@yahoo.com \\ ${ }^{3}$ Young Researchers Club, Islamic Azad University, Ayatollah Amoli Branch, Amol, Iran, \\ Edrishendoii@gmail.com
}

Article history:

Received February 2013

Accepted March 2013

Available online April 2013

\begin{abstract}
In this paper,introduce the concept of normal fuzzy subhypernear-modules of hypernear-modules and establish three isomorphism theorems of hypernear-modules by using normal fuzzy subhypernear-modules.
\end{abstract}

Keywords: Near-module, Hypernear-module, Normal fuzzy subhypernear-module, Isomorphism theorems

\section{Introduction}

Hyperstructures, in particular hypergroups, were introduced in 1934 by a French mathematician, Marty, at the VIIIth Congress of Scandinavian Mathematicians ([20]). Since then, hundreds of papers and several books have been written on this topic. Nowadays, hyperstructures have a lot of applications to several domains of mathematics and computer science see $[1,2,4,6,7,9,13]$, and they are studied in many countries of Europe, America and Asia. In 1971, Rosenfeld [23] introduced fuzzy sets in the context of group theory and formulated the concept of a fuzzy subgroup of a group. Since then, many researchers are engaged in extending the concepts of abstract algebra to the framework of the fuzzy setting. In 1990 Dasic [10] has introduced the notation of hypernear-rings in a particular case. The hypernear-rings generalize the concept of near-ring. More recently, Sen, Ameri and Chowdhury introduced and analyzed fuzzy semihypergroups in [24]. The fuzzy hyperring notion is defined and studied in [17].Ameri and Hendoukolaie introduced and analyzed fuzzy hypernear-ring and a fuzzy hypernear-module on a hypernear-ring in [2,3]. in [14] Hendukolaie analyzed the fuzzy homomorphism between Hypernear-rings and in [15] Hendukolaie, Ghasemi, Ghasemi introduced and analized the fuzzy isomorphism theorem of $\Gamma$-hypernear-rings by $\Gamma$ -hyperideals. J. Zhan, B. Davvaz, K.P. Shum, introduced the concept of normal fuzzy 
subhypermodules of hypermodules and analized three isomorphism theorems of hypermodules by using normal fuzzy subhypermodules in [29]. In this paper, introduce the concept of normal fuzzy subhypernear-modules of hypernear-modules and establish three isomorphism theorems of hypernear-modules by using normal fuzzy subhypernear-modules.

\section{Preliminaries}

First of all,Recalled some notions and results that used in the following paragraphs. ( see [1],[5],[6],[20]). A nonempty set $R$ with two binary hyperoperations " · and " + is called a Near-ring if:

(i) $(R,+)$ is a group;

(ii) $(R, \cdot)$ is a semigroup;

(iii) $x \cdot(y+z)=x \cdot y+x \cdot z, \quad \forall x, y, z \in R$.

Definition 2.1 A right $R$-nearmodule $M$ over a Near-ring $R$ consists of an group $(M,+)$ and an operation $M \times R \rightarrow M$ such that for all $x, y$ of $M$ and $r, s$ of $R$, We have:

(i) $(x+y) . r=x \cdot r+y \cdot r$;

(ii) $x .(r+s)=x . r+x . s$;

(iii) $x .(r . s)=(x . r) . s$;

(iv) $x .1_{R}=x$ if $R$ has multiplicative identity $1_{R}$.

Example 2.2 every module $M$ over a ring $R$ is a near-module.

Example 2.3 If $K$ is a field, Then the concepts $K$-vectorspace (a vector space over $K$ ) and $K$-nearmodule are identical.

Let $H$ be a nonempty set and let $P^{*}(H)$ be the set of all nonempty subsets of $H$. A hyperoperation on $H$ is a map $\circ: H \times H \rightarrow P^{*}(H)$ and the couple $(H, \circ)$ is called a hypergroupoid.

If $A$ and $B$ are nonempty subsets of $H$, then we denote

$$
A \circ B=\bigcup_{a \in A, b \in B} a \circ b, \quad A \circ x=A \circ\{x\}, \quad x \circ B=\{x\} \circ B .
$$

A hypergroupoid $(H, \circ)$ is called a semihypergroup if for all $x ; y ; z$ of $H$ we have $(x \circ y) \circ z=x \circ(y \circ z)$, which means that

$$
\bigcup_{u \in x \circ y} u \circ z=\bigcup_{v \in y \circ z} x \circ v \text {. }
$$

An element $e$ of $H$ is called an identity (scalar identity) of $(H, \circ)$ if for all $a \in H$, we have $a \in(e \circ a) \cap(e \circ a), \quad(\{a\}=(e \circ a) \cap(e \circ a))$.

A hypergroup is a semihypergroup such that for all $x \in H$, we have $x \circ H=H=H \circ x$.

A subhypergroup $(K, \circ)$ of $(H, \circ)$ is a nonempty set $K$, such that for all $k \in K$, we have $k \circ K=K=k \circ K$.

Definition 2.4 The triple $(R,+$,$) is a hypernear-ring if:$ 
(1) $(R, \quad+)$ is a quasicanonical hypergroup, i.e. the following axioms hold for $(R, \quad+)$ :

(i) $(x+y)+z=x+(y+z), \quad \forall x, y, z \in R$;

(ii) $\exists 0 \in R$ such that $x+0=x=0+x, \quad \forall x \in R$;

(iii) $\forall x \in H, \quad \exists ! x^{\prime} \in H$ such that $0 \in\left(x+x^{\prime}\right) \cap\left(x^{\prime}+x\right)$;

(iv) $\forall x, y, z \in R$ and $z \in x+y \Rightarrow x \in z+(-y), \quad y \in(-x)+z$.

(2) $(R, \cdot)$ is a semihypergroup having 0 as a right absorbing element, i.e. $0 \cdot x=0$, $\forall x \in R$

(3) $(x+y) \cdot z=x \cdot z+y \cdot z, \quad \forall x, y, z \in R$.

Let $(R,+$,$) be a hypernear-ring . A non-empty subset A$ of $R$ is called a subhypernear-ring of $R$ if $(A,+$,$) itself a hypernear-ring. A subhypernear-ring A \subseteq R$ is called normal if for all $x \in R$ holds:

$$
x+A-x \subseteq A \text {. }
$$

Since $A \subseteq x+A-x$, it follows $A=x+A-x$, for all $x \in R$.

Definition 2.5 Let $(R,+, \cdot)$ be a hypernear-ring. A nonempty set $M$, endowed with two hyperoperations $\oplus$, e is called a right hypernear-module over $(R,+, \cdot)$ if the following conditions hold:

(1) $(M, \oplus)$ is a hypergroup ( not necessarily commutative).

(2) $\mathrm{e}: M \times R \rightarrow P^{*}(M)$ is such that for all $a, b$ of $M$ and $r, s$ of $R$, we have:

(i) $(a \oplus b) \mathrm{e} r=(a \mathrm{e} r) \oplus(b \mathrm{e} r)$;

(ii) $a \mathrm{e}(r+s)=(a \mathrm{e} r) \oplus(a \mathrm{e} s)$;

(iii) $a \mathrm{e}(r . s)=(a \mathrm{e} r) \mathrm{e} s$;

(iv) $a \mathrm{e} 0=0$ and $0 . r=0$.

Let $(M, \oplus, \mathrm{e})$ be a hypernear-module. A non-empty subset $A$ of $M$ is called a subhypernear-module of $(M, \oplus, \mathrm{e})$ if $(A, \oplus, \mathrm{e})$ itself a hypernear-module.

A subhypernear-module $\mathrm{A}$ of $\mathrm{M}$ is called normal if the relation $x+A-x \subseteq A$ holds for all $x \in M$.

Example 2.6 Every right hypermodule $M$ over a hyperring $R$ is a right hypernear-module.

Example 2.7 Let $(R,+)$ be a hypergroup ( not necessarily commutative) and let $\left(M_{0}(R),+, \circ\right)$ be a hypernear-ring of mapping from $R$ into itself (see[8]). Then $(R, \oplus, \mathrm{e})$ be a hypernear-ring over $\left(M_{0}(R),+, \circ\right)$, Where the action $\mu: R \times M_{0}(R) \rightarrow R$ is given by $(a, f) \rightarrow(a) f$, for all $a \in R$ and $f \in M_{0}(R)$.

Let $\mathrm{A}$ be a subhypernear-module of an R-hypernear-module $\mathrm{M}$. Then the hyperquotient group $M / A=\{m+A \mid m \in M\} \quad$ endowed with the following external composition $M / A \times R \rightarrow M / A,(m+A, r) \rightarrow m r+A$, is an R-hypernear-module, and $M / A$ is called the quotient R-hypernear-module of $\mathrm{M}$ by $\mathrm{A}$.

In what follows, all the hypernear-modules are right hypernear-modules.

Definition 2.8 A fuzzy subset $\mu$ of a hypernear-module $M$ over a hypernear-ring $R$ is called $a$ 
fuzzy subhypernear-module of $M$ if the following conditions hold:

(i) $\min \{\mu(x), \mu(y)\} \leq \inf _{z \in x+y} \mu(z)$, for all $x, y \in M$;

(ii) $\mu(x) \leq \mu(-x)$, for all $x \in M$;

(iii) $\mu(x) \leq \mu(x . r)$, for all $r \in R$ and $x \in M$.

A fuzzy subhypernear-module $\mu$ of $\mathrm{M}$ is called normal if $\mu(y) \leq \inf f_{z \in x+y-x} \mu(z)$, for all $x, y \in M$.

If $\mu$ be a fuzzy subhypernear-module of $\mathrm{M}$, then it is clear that $\mu(-x)=\mu(x)$, $\min \{\mu(x), \mu(y)\} \leq \inf f_{z \in x-y} \mu(z)$, for all $x, y \in M$.

Let $\mathrm{M}$ be an R-hypernear-module. Then, for a fuzzy subset $\mu$ of $\mathrm{M}$, the level subset $\mu_{t}$ and the strong level subset $\mu_{t}^{>}$are defined by

$$
\mu_{t}=\{x \in M \mid \mu(x) \geq t\}, t \in[0,1]
$$

and

$$
\mu_{t}^{>}=\{x \in M \mid \mu(x)>t\}, t \in[0,1] .
$$

A fuzzy subhypernear-module can be characterized by using its level subsets and strong level subsets. The following proposition is obvious.

Proposition 2.9 Let $\mu$ be a fuzzy subset of an R-hypernear-module $M$. Then the following statements are equivalent:

(1) $\mu$ is a fuzzy subhypernear-module of $M$,

(2) each non-empty strong level subset of $\mu$ is a subhypernear-module of $M$,

(3) each non-empty level subset of $\mu$ is a subhypernear-module of $M$.

Definition 2.10 A mapping $f: M \rightarrow M^{\prime}$ is called a homomorphism if for all $a, b \in M$ and $r \in R$, we have:

$$
f(a+b)=f(a)+f(b), \quad f(a . r)=f(a) . r \text { and } f(0)=0
$$

It is clear that a homomorphism $\mathrm{f}$ is an isomorphism if $\mathrm{f}$ is both injective and surjective and write $M \cong M^{\prime}$ if $\mathrm{M}$ is isomorphic to $M^{\prime}$.

\section{The isomorphism theorem}

In what follows, $\mathrm{M}$ is always a hypernear-module over a hypernear-ring $\mathrm{R}$ unless state otherwise.

Definition 3.1 Let $\mu$ be a normal fuzzy subhypernear-module of $M$. Define the following relation on $M$.

$x \equiv y(\bmod \mu) \quad$ if and only if there exists $\alpha \in(x-y)$ such that $\mu(\alpha)=\mu(0)$.

now denote the above relation by $x \mu^{*} y$. Then, for this relation, we have the following lemma.

Lemma 3.2 The relation $\mu^{*}$ is an equivalence relation.

Proof. For all $x, y, z \in M$, we have

(i) $0 \in x-x$ implies $x \mu^{*} x$, i.e., $\mu^{*}$ is reflexive;

(ii) if $x \mu^{*} y$ then there exist $\alpha \in(x-y)$ such that $\mu(\alpha)=\mu(0)$. Since 
$\mu(\alpha)=\mu(-\alpha)$ and $-\alpha \in(y-x), y \mu^{*} x$. Thus, $\mu^{*}$ is symmetric.

(iii) To prove that $\mu^{*}$ is transitive, let $x \mu^{*} y$ and $y \mu^{*} z$. Then there exist then there exist $\alpha \in(x-y)$ and $\beta \in(y-z)$ such that $\mu(\alpha)=\mu(\beta)=\mu(0)$. Therefore, $x \in \alpha+y$ and $-z \in y+\beta$. Hence, we have $-z+x \subseteq-y+\beta+\alpha+y$, and so for every $a \in-z+x$, there exists $b \in \beta+\alpha$ such that $a \in-y+b+y$. Since $\mu$ is normal, $\mu(b) \leq \mu(a)$ and $\mu(0)=\min \{\mu(\alpha), \mu(\beta)\} \leq \mu(b)$. These imply that $\mu(b)=\mu(0)$. Consequently, we have $a \in-z+x$ and $\mu(a)=\mu(0)$, and so $(-z) \mu^{*}(-x)$, that is, $x \mu^{*} z$. This completes the proof.

Lemma 3.3 If $x \mu^{*} y$, then $\mu(x)=\mu(y)$.

Proof. if $x \mu^{*} y$ then there exist $\alpha \in x-y$ such that $\mu(\alpha)=\mu(0)$. Since $\alpha \in x-y$ implies $x \in \alpha+y$ and so $\min \{\mu(\alpha), \mu(y)\} \leq \mu(x)$, that is, $\mu(y) \leq \mu(x)$. Similarly, we have $\mu(x) \leq \mu(y)$. Hence $\mu(x)=\mu(y)$.

Let $v$ be an equivalence relation on $\mathrm{M}$. If $A, B$ are non-empty subsets of $\mathrm{M}$, then we write $A \bar{v} B$ to denote that

$\forall a \in A, \exists b \in B$ such that $a v b$ and

$\forall b \in B, \exists a \in A$ such that $a v b$.

An equivalence relation $v$ on $\mathrm{M}$ is called regular if for every $x, y \in M$,

$x y y \Rightarrow x+z \overline{v y}+z$, for all $z \in M$.

Lemma $3.4 \mu^{*}$ is a regular relation.

Proof. Suppose that $x \mu^{*} y$. Then there exists $\alpha^{\prime} \in x-y$ such that $\mu\left(\alpha^{\prime}\right)=\mu(0)$. Now, for every $z \in M$ and $a \in x+z$, we have $x \in a-z$ which implies that $x-y \subseteq a-z-y$ or $x-y \subseteq a-(y+z)$. Hence $\alpha^{\prime} \in a-(y+z)$ and so there exists $b \in y+z$ such that $\alpha^{\prime} \in a-b$. Thus, $a \mu^{*} b$ and so $(x+z) \bar{\mu}^{*}(y+z)$.

Let $\mu^{*}[x]$ be the equivalence class containing the element $\mathrm{x}$. Then we denote $M / \mu$ the set of all equivalence classes, i.e., $M / \mu=\left\{\mu^{*}[x] \mid x \in M\right\}$. Define the following two operations on $M / \mu$ :

$$
\begin{aligned}
& \mu^{*}[x]\left(\mu^{*}[y]=\left\{\mu^{*}[z] \mid z \in \mu^{*}[x]+\mu^{*}[y]\right\}\right. \\
& \mu^{*}[x]^{*} r=\mu^{*}[x . r] .
\end{aligned}
$$

Since $\mu^{*}$ is regular, we can easily deduce the following theorem:

Theorem $3.5\left(M / \mu,\left(,^{*}\right)\right.$ is a hypernear-module.

Let $f: M \rightarrow M^{\prime}$ be a map and $\mu, \lambda$ be the fuzzy subsets of $\mathrm{M}, M^{\prime}$ respectively. Then the image $f(\mu)$ of $\mu$ is the fuzzy subset of $\mathrm{M}$ defined by

$$
f(\mu)(y)=\left\{l l \text { sup_x } f^{\wedge}-1(y)\{(x)\} \quad \text { if } \quad f^{\wedge}-1(y) \quad 0\right. \text { otherwise.. }
$$


for all $y \in M^{\prime}$. The inverse image $f^{-1}(\lambda)$ of $\lambda$ is the fuzzy subset of $M$ defined by $f^{-1}(\lambda)(x)=\lambda(f(x))$ for all $x \in M$. The following two lemmas can be easily proved and hence, we omit the details.

Lemma 3.6 Let $f: M \rightarrow M^{\prime}$ be a homomorphism of hypernear-modules and $\mu$ a (normal) fuzzy subhypernear-module of $M$. Then $f(\mu)$ is a (normal) fuzzy subhypernear-module of $M^{\prime}$

Lemma 3.7 Let $f: M \rightarrow M^{\prime}$ be a homomorphism of hypernear-modules and $\mu, \lambda$ a normal fuzzy subhypernear-module of $M, M^{\prime}$, respectively. Then, the following statements hold:

(i) If $\mathrm{f}$ is an epimorphism, then $f\left(f^{-1}(\lambda)\right)=\lambda$;

(ii) If $\mu$ is a constant on $\operatorname{Ker} \mathrm{f}$, then $f^{-1}(f(\mu))=\mu$.

Let $\mu$ be a normal subhypernear-module of $\mathrm{M}$. We now denote $M_{\mu}=\{x \in M \mid \mu(x)=\mu(0)\}$. Clearly, $M_{\mu}$ is a normal subhypernear-module of $\mathrm{M}$. We now use the normal subhypernear-module of $\mathrm{M}$ to establish the isomorphism theorems.

Theorem 3.8 (First fuzzy isomorphism theorem) Let $f: M \rightarrow M^{\prime}$ be an epimorphism of hypernear-modules and $\mu$ a normal fuzzy subhypernear-module of $M$ with $M_{\mu} \supseteq$ Kerf. Then $M / \mu \cong M^{\prime} / f(\mu)$.

Proof. First note that $M / \mu$ and $M^{\prime} / f(\mu)$ are hypernear-modules. Now, Define $\varphi: M / \mu \rightarrow M^{\prime} / f(\mu)$ by $\varphi\left(\mu^{*}[x]=f(\mu)^{a}[f(x)]\right.$, for all $x \in M$. Then $\varphi$ is clearly well-defined. In fact, if $\mu^{*}[x]=\mu^{*}[y]$, then $\mu(x)=\mu(y)$ by Lemma 3.3. Since $M_{\mu} \supseteq \operatorname{Kerf}$, $\mu$ is a constant on Kerf . By Lemma 3.7(ii), we have $f^{-1}(f(\mu))=\mu$. . Thus, $f^{-1}(f(\mu))(x)=f^{-1}(f(\mu))(y)$. It follows from above the definition that $f(\mu)(f(x))=f(\mu)(f(y))$. Hence we $f(\mu)^{\mathrm{a}}[f(x)]=f(\mu)^{\mathrm{a}}[f(y)]$. Moreover, we have

$$
\begin{aligned}
& \varphi\left(\mu^{*}[x]\left(\mu^{*}[y]\right)=\varphi\left(\left\{\mu^{*}[z] \mid z \in \mu^{*}[x]+\mu^{*}[y]\right\}=\left\{f(\mu)^{*}[f(z)] \mid z \in \mu^{*}[x]+\mu^{*}[y]\right\}\right.\right. \\
& =f(\mu)^{*}\left(f\left(\mu^{*}[f(x)]\right)\right)+f(\mu)^{*}\left(f\left(\mu^{*}[f(y)]\right)\right)=\varphi\left(\mu^{*}[x]\right)\left(\varphi\left(\mu^{*}[y]\right) ;\right.
\end{aligned}
$$

(ii) $\varphi\left(\mu^{*}[x]^{*} r\right)=\varphi\left(\mu^{*}[x . r]\right)=f(\mu)^{*}(f(x . r))=f(\mu)^{*}(f(x) . r)=f(\mu)^{*}([f(x)])^{*} r=\varphi\left(\mu^{*}[x]^{*} r\right.$. (iii) $\varphi\left(\mu^{*}[0]\right)=f(\mu)^{\stackrel{a}{a}}[f(0)]=f(\mu)^{a}[0]=0$.

Hence, we have shown that $\varphi$ is a homomorphism. Clearly $\varphi$ is an epimorphism. To show that $\varphi$ is a monomorphism, Let $f(\mu)^{\mathrm{a}}[f(x)]=f(\mu)^{\mathrm{a}}[f(y)]$. Then $f(\mu)(f(x))=f(\mu)(f(y))$, that is $f^{-1}(f(\mu))(x)=f^{-1}(f(\mu))(y)$, Hence $\mu(x)=\mu(y)$, and so $\mu^{*}[x]=\mu^{*}[y]$, therefore, $M / \mu \cong M^{\prime} / f(\mu)$.

Lemma 3.9 Let $f: M \rightarrow M^{\prime}$ be an epimorphism of hypernear-modules. If $\lambda$ be a (normal) fuzzy subhypernear-module of $M^{\prime}$, then $f^{-1}(\lambda)$ is a (normal) fuzzy subhypernear-module of $M$.

Corollary 3.10 Let $f: M \rightarrow M^{\prime}$ be an epimorphism of hypernear-modules. If $\lambda$ be a normal fuzzy subhypernear-module of $M^{\prime}$, then $M / f^{-1}(\lambda) \cong M^{\prime} / \lambda$ 
Proof. First we observe that $M / f^{-1}(\lambda)$ and $M^{\prime} / \lambda$ are hypernear-modules by Lemma 3.9. In order to prove that $M_{f^{-1}(\lambda)} \supseteq k e r f$, we consider $x \in \operatorname{Kerf}$. Then we have $f(x)=f(0)$, and hence $\lambda(f(x))=\lambda(f(0))$, i.e., $f^{-1}(\lambda)(x)=f^{-1}(\lambda)(0)$, This leads to $x \in M_{f^{-1}(\lambda)}$, and so $M_{f^{-1}(\lambda)} \supseteq k \operatorname{kerf}$. By Theorem 3.8, we have $M / f^{-1}(\lambda) \cong M^{\prime} / \lambda$.

Now, we proceed to establish the Second and Third Fuzzy Isomorphism Theorems. The following two lemmas are obvious.

Lemma 3.11 Let $A$ be a normal subhypernear-module of $M$ and $\mu$ a normal fuzzy subhypernear-module of $M$. Then the following statements hold:

(i) If $\mu$ is restricted to $A$, then $\mu$ is a normal fuzzy subhypernear-module of $A$;

(ii) $A / \mu$ is a normal subhypernear-module of $M / \mu$.

Lemma 3.12 If $\mu$ and $\lambda$ are any two normal fuzzy subhypernear-modules of $M$, then so is $\mu \cap \lambda$.

We now prove our second fuzzy isomorphism theorem:

Theorem 3.13 (Second fuzzy isomorphism theorem) If $\mu$ and $\lambda$ are any two normal fuzzy subhypernear-modules of $M$ with $\mu(0)=\lambda(0)$, then,

$$
M_{\mu} /(\mu \cap \lambda) \cong\left(M_{\mu}+M_{\lambda}\right) / \lambda
$$

Proof. By Lemmas 3.11 and 3.12, $\lambda$ and $\mu \cap \lambda$ are two normal fuzzy subhypernear-modules of $M_{\mu}+M_{\lambda}$ and $M_{\mu}$, respectively. Now, it is clear that $\left(M_{\mu}+M_{\lambda}\right) / \lambda$ and $M_{\mu} /(\mu \cap \lambda)$ are both hypernear-modules. Define $\psi: M_{\mu} \rightarrow\left(M_{\mu}+M_{\lambda}\right) / \lambda$ by $\psi(x)=\lambda^{a}[x]$, for all $x \in M_{\mu}$. Then, it is easy to check that $\psi$ is an epimorphism. To show that $\operatorname{Ker} \psi=M_{\mu \cap \lambda}$. we consider the following equalities:

$\operatorname{Ker} \psi=\left\{x \in M_{\mu} \mid \psi(x)=\lambda^{a}[0]\right\}=\left\{x \in M_{\mu} \mid \lambda^{a}[x]=\lambda^{a ̊}[0]\right\}=$

$\left\{x \in M_{\mu} \mid \lambda(x)=\lambda(0)\right\}=\left\{x \in M_{\mu} \mid \mu(x)=\mu(0)=\lambda(0)=\lambda(x)\right\}$

$=\left\{x \in M_{\mu} \mid x \in M_{\lambda}\right\}=M_{\mu \cap \lambda}$

Therefore, $M_{\mu} /(\mu \cap \lambda) \cong\left(M_{\mu}+M_{\lambda}\right) / \lambda$.

Theorem 3.14 (Third fuzzy isomorphism theorem) Let $\mu$ and $\lambda$ are any two normal fuzzy subhypernear-modules of $M$ with $\mu \geq \lambda$ and $\mu(0)=\lambda(0)$. then,

$$
(M / \lambda) /\left(M_{\mu} / \lambda\right) \cong M / \mu
$$

Proof. By Lemma 3.11(ii), it is known that $M_{\mu} / \lambda$ is a normal subhypernear-module of $M / \lambda$. Define $f: M / \lambda \rightarrow M / \mu$ by $f\left(\lambda^{a}[x]\right)=\mu^{a}[x]$, for all $x \in M$. If $\lambda^{a}[x]=\lambda^{a}[y]$, for all $x, y \in M$, then there exists $\alpha \in x-y$ such that $\lambda(\alpha)=\lambda(0)$. Since $\mu \geq \lambda$ and $\mu(0)=\lambda(0)$, 
we have $\mu(\alpha) \geq \lambda(\alpha)=\lambda(0)=\mu(0)$. This implies that $\mu(\alpha)=\mu(0)$, and so $\mu^{a}[x]=\mu^{a ̊}[y]$. Hence, $\mathrm{f}$ is well-defined. Moreover, we have

(i) $f\left(\lambda^{\mathrm{a}}[x]\left(\lambda^{\mathrm{a}}[y]\right)\right.$

$=f\left(\left\{\lambda^{\mathrm{a}}[z] \mid z \in \lambda^{\mathrm{a}}[x]+\lambda^{\mathrm{a}}[y]\right\}\right)$

$=\left\{\mu^{\mathrm{a}}[z] \mid z \in \lambda^{\mathrm{a}}[x]+\lambda^{\mathrm{a}}[y]\right\}$

$=\mu^{a ̊ a}\left[\lambda^{a}[x]\right]\left(\mu^{a}\left[\lambda^{a}[y]\right]\right.$

$=\mu^{\stackrel{a}{\mathrm{a}}}[x]\left(\mu^{\mathrm{a}}[y]\right.$

$=f\left(\lambda^{a}[x]\right)\left(f\left(\lambda^{a}[y]\right)\right.$

(ii) $f\left(\lambda^{\mathrm{a}}[x]^{\star} r\right)=f\left(\lambda^{\mathrm{a}}[x . r]\right)=\mu^{\mathrm{a}}[x . r]=\mu^{\mathrm{a}}[x]^{\star} r=f\left(\lambda^{\mathrm{a}}[x]\right)^{*} r$,

(iii) $f\left(\lambda^{\mathrm{a}}[0]\right)=\mu^{\mathrm{a}}[0]=0$.

Hence, $\mathrm{f}$ is a homomorphism. Clearly, $\mathrm{f}$ is an epimorphism. Now we show that $\operatorname{Kerf}=M_{\mu} / \lambda$. In fact

$$
\begin{aligned}
& \text { kerf }=\left\{\lambda^{a}[x] \in M / \lambda \mid f\left(\lambda^{a ̊ a}[x]\right)=\mu^{\mathrm{a}}[0]\right\} \\
& =\left\{\lambda^{a}[x] \in M / \lambda \mid \mu^{\mathrm{a}}[x]=\mu^{\mathrm{a}}[0]\right\} \\
& =\left\{\lambda^{a}[x] \in M / \lambda \mid \mu[x]=\mu[0]\right\} \\
& =\left\{\lambda^{a}[x] \in M / \lambda \mid x \in M_{\mu}\right\} \\
& =M_{\mu} / \lambda .
\end{aligned}
$$

Therefore, $(M / \lambda) /\left(M_{\mu} / \lambda\right) \cong M / \mu$.

\section{References}

[1] R. Ameri, On categories of hypergroups and hypermodules. J Discrete Math Sci Cryptogr 6(2-3): 121-132 (2003).

[2] R. Ameri, E. Hendoukolaii, Fuzzy Hypernear-rings, to appear.

[3] R. Ameri, E. Hendoukolaii, Fuzzy Hypernear-modules, to appear.

[4] P. Corsini, Fuzzy sets, join spaces and factor spaces, PU.M.A. 11 (3) 439446 (2000).

[5] P. Corsini, V. Leoreanu, Fuzzy sets and join spaces associated with rough sets, Circ. Mat. Palermo 51527536 (2002).

[6] P. Corsini, V. Leoreanu, Join spaces associated with fuzzy sets, J. Combin. Inform. System Sci. 20 (1 4) 293303 (1995).

[7] P. Corsini, Prolegomena of Hypergroup Theory, second ed., Aviani Editor, (1993).

[8] P. Corsini, V. Leoreanu, Applications of Hyperstructures Theory, Advanced in Mathematics, Kluwer Academic Publishers, (2003).

[9] P. Corcini, I. Tofan, On fuzzy hypergroups, P.U.M.A. (8), 29-37 (1997).

[10] V. Dasic, Hypernear-rings, in: Proc. Fourth Int. Congress on AHA, World Scientific, 1991, pp. $75-85$ (1990).

[11] B. Davvaz, Fuzzy Hv-groups, Fuzzy Sets Syst. 101, 191195 (1999).

[12] B. Davvaz, Fuzzy Hv submodules, Fuzzy Sets Syst. 117, 477484 (2001).

[13] V.M. Gontineac, On hypernear-rings and H-hypergroups, in: Proc. Fifth Int. Congress on 
AHA, Hadronic Press Inc., USA, (1994), 171-179 (1993).

[14] E. Hendukolaie, On fuzzy homomorphisms between Hypernear-rings, The journal of mathematics and computer science, vol.2, num.4, 702-716 (2011).

[15] E. Hendukolaie, A.A. Ghasemi, G. Ghasemi, On fuzzy isomorphisms theorems of $\Gamma$ -Hypernear-rings, to appear.

[20] V. Leoreanu-Fotea, B. Davvaz, n-Hypergroups and binary relations, European J. Combin. 29, 12071218 (2008).

[17] V. Leoreanu-Fotea, B. Davvaz, Fuzzy hyperrings,Fuzzy Sets and Systems, doi: 10.1016/j.fss.2008.11.007 (2009).

[18] V. Leoreanu-Fotea, B. Davvaz, Fuzzy hypermodules, Computers and Mathematics with Applications 57, 466475 (2009).

[19] V. Leoreanu-Fotea, B. Davvaz, Join n-spaces and lattices, Multiple Valued Logic Soft Comput. 15, accepted for publication (2008).

[20] F. Marty, Sur une généralisation de la notion de group, in: 4th Congress Math. Scandinaves, Stockholm, pp. 4549 (1934).

[21] J.N. Mordeson, M.S. Malik, Fuzzy Commutative Algebra, Word Publ., (1998).

[22] W. Prenowitz, J. Jantosciak, Join Geometries, Springer UTM, (1979).

[23] A. Rosenfeld,Fuzzygroups, J. Math. Anal. Appl. 35, 512-517 (1971).

[24] M.K. Sen, R. Ameri, G. Chowdhury, Fuzzy hypersemigroups, Soft Comput., doi: http://10.1007/s00500-007-0257-9 (2007).

[25] T. Vougiouklis, Hyperstructures and Their Representations, Hadronic Press Inc., Palm Harber, p. 115 (1994).

[26] T. Vougiouklis, Fundamental relations in hyperstructures, Bull. Greek Math. Soc. 42, 113118 (1999).

[27] Zhan J, Davvaz B, Shum KP A new view of fuzzy hyper- modules. Acta Math Sin Engl Ser 23(4) (2007b).

[28] Jianming Zhan. Bijan Davvaz- K. P. Shum, On fuzzy isomorphism theorems of hypermodules, Soft Comput, 11:1053-1057 DOI 10.1007/s00500-007-0152-4 (2007).

[29] M.M. Zahedi, R. Ameri, On the prime, primary and maximal subhypermodules, Ital. J. Pure Appl. Math. 5, 61-80 (1999). 